\title{
Cognitive Deficits Caused by Late Gestational Disruption of Neurogenesis in Rats: a Preclinical Model of Schizophrenia
}

\author{
Peter Flagstad*,', Birte Y Glenthøj ${ }^{2}$ and Michael Didriksen' \\ 'Department of Pharmacology Target Research, H Lundbeck A/S, Copenhagen-Valby, Denmark; '2Department of Psychiatry E, Copenhagen \\ University Hospital, Bispebjerg, Denmark
}

\begin{abstract}
Late gestational disruption of neurogenesis in rats has been shown to induce behavioral abnormalities thought to mimic aspects of positive and negative symptoms of schizophrenia. Furthermore, it has been shown that the morphological changes produced by the perturbation are relevant to schizophrenia with reduced thickness of the hippocampus, thalamus, and cortical regions. In addition to the positive and negative symptoms, schizophrenia is associated with deficits in a wide variety of cognitive domains. In the present studies, we assessed whether the cognitive deficits are modeled by disruption of neurogenesis late during gestation (gestational day 17) in the rat. In the battery of tests utilized, we describe that rats in which neurogenesis was disrupted have deficits in a reversal-learning paradigm of the Morris water maze and in object recognition, and that they exhibit perseveration in the Porsolt forced swimming test. Additionally, we found deficient associative learning in an acquisition of an active avoidance paradigm and deficits in latent inhibition. No deficits were observed in the reference memory version of the Morris water maze and in a non-match-to position experiment, showing that the deficits are limited to certain aspects of cognition.

Neuropsychopharmacology (2005) 30, 250-260, advance online publication, I December 2004; doi: I 0. I 038/sj.npp. I 300625
\end{abstract}

Keywords: schizophrenia; cognition; animal models; methylazoxymethanol; prenatal; neurodevelopment

\section{INTRODUCTION}

The cytoarchitectural and morphological brain changes observed in schizophrenia are consistent with a pre/ perinatal origin (Kovelman and Scheibel, 1984; Conrad et al, 1991; Jakob and Beckmann, 1986; Arnold et al, 1991). This, along with epidemiological data (Buka et al, 2001; Zornberg et al, 2000; Susser et al, 1996; Mednick et al, 1988), has led to the neurodevelopmental theory of schizophrenia, suggesting that neurodevelopmental disturbances contribute to the etiology of at least some cases of the disorder. The disturbance in brain development can be caused either by genetic factors or by external factors, such as prenatal exposure to influenza virus, birth complications, etc. It has previously been shown in preclinical models that disturbance of neurodevelopment in rats can cause behavioral changes relevant to positive as well as negative symptoms of schizophrenia (Flagstad et al, 2004; Lipska et al, 1993; Sams-Dodd et al, 1997; Le Pen et al, 2002; Daenen et al, 2002; Talamini et al, 1999). One way of

* Correspondence: P Flagstad, Department of Pharmacology Target Research, H Lundbeck A/S, Ottiliavej 9, DK-2500 Copenhagen-Valby, Denmark, Tel: + 45 36433047, Fax: + 45 36438258,

E-mail: PFLA@Lundbeck.com

Received 27 July 2004; revised 5 October 2004; accepted 21 October 2004

Online publication: 25 October 2004 at http://www.acnp.org/citations/ Npp I02504040343/default.pdf inducing prenatal disturbance is by treatment with the mitosis inhibitor methylazoxymethanol (MAM) during gestation (Johnston et al, 1988; Johnston and Coyle, 1982; Flagstad et al, 2004; Balduini et al, 1991; Virgili et al, 1989). Specifically, treatment on gestational day 17 (GD17) has proven to produce morphological changes consistent with findings in schizophrenia (Moore and Grace, 1997; Flagstad et al, 2004), as well as behavioral changes mimicking the positive and negative symptoms of the disorder (Flagstad et al, 2004).

Schizophrenia is associated with deficits in a wide variety of cognitive domains including executive function, attention, memory, and language (Goldberg and Gold, 1995). Cognitive impairments are believed to be the core symptom of the disease. It has been proposed that a disturbance of an underlying core cognitive process could cause the deficits in the many different aspects of cognition (Hemsley, 1994; Andreasen, 1999); this subject, however, remains controversial because of the heterogeneity of the clinical symptoms that characterize the disorder. Another way of mapping the cognitive deficits is to locate structures in which abnormal morphology correlates with a deficient cognitive process or which has altered activation during cognitive processes. These studies have provided evidence that the deficient cognitive processes are associated with dysfunctions within the frontotemporal cortex. Evidence of abnormal frontal lobe activation includes investigations using prefrontal-dependent tasks of working memory and 
executive functions like the Wisconsin Card Sorting Test, a prefrontal-dependent test of attentional set shifting abilities (Berman et al, 1992), or the 'Tower of London', which tests the ability to create strategies and plan how they are implemented (spontaneous flexibility) (Andreasen et al, 1992). The temporal lobe has likewise been shown to have metabolic abnormalities during memory tasks (Gur et al, 1994; Heckers et al, 1998). Furthermore, evidence suggests that the metabolic abnormalities might not be confined to a single area, but rather a network of interconnected areas (Andreasen et al, 1998; Ragland et al, 1998, 2001; Buchsbaum et al, 1992).

As the MAM GD17 model of schizophrenia has so far been shown to have good homology to positive as well as negative schizophrenic symptoms, it is of interest to investigate possible changes in cognitive capacity. A previous study has shown that rats treated on embryonic day 17 have deficits in an eight-arm radial maze task (Gourevitch et al, 2004). Furthermore, electrophysiological studies performed on MAM-treated rats indicate deficient hippocampal gating of the nucleus accumbens, suggesting perhaps an inability to use context to guide behavior (Grace, 2000, 2003; Grace and Moore, 1998).

Cognitive deficits in memory, executive function, and working memory are believed to be of major importance to the functional and symptomatic outcome (Fujii and Wylie, 2003; Moritz et al, 2000; Grawe and Levander, 2001; Green et al, 2000) as well as the quality of life (Fujii et al, 2004) of patients with schizophrenia. Being able to address some of these symptoms in a preclinical model and consequently develop drugs that better target these symptoms could lead to better outcome and better quality of life for the patients.

In the present experiments, we used a battery of tests to assess cognition in rats with a MAM GD17-induced neurodevelopmental disturbance. The tests include two versions of the Morris water maze, a delayed non-match-toposition (DNMTP) paradigm, a forced swimming test, an object recognition test, and finally we assessed associative learning and latent inhibition in a two-way shuttle box.

\section{MATERIALS AND METHODS}

\section{Animals}

Pregnant (Wistar, Brl) dams were obtained from the animal provider (M\&B, Denmark) on gestational day (GD) 10 and housed individually in polycarbonate tubs. On GD 17, the dams were treated with $22 \mathrm{mg} / \mathrm{kg}$ MAM or saline i.p. Within 4 days after birth, the litters were culled to 10. Pups were weaned on day 30 and housed in pairs. Only male rats were included in the studies.

The rats were housed in an animal room in macrolon type III cages. The animal room was kept at $21 \pm 2^{\circ} \mathrm{C}$ with a relative humidity of $55 \pm 5 \%$. The rats were kept on a normal light/dark cycle (light on 0600-1800) with free access to water and commercial food pellets. They were moved to the experimental room on the day before testing. Experiments were conducted on offspring between postnatal days 80 and 120, except in the DNMTP experiment where animals were 60 days old when the training began (total period of experiment $\sim 5$ months). In all the experiments, animals were randomly assigned from a large number of litters (the number of litters were always larger than the number of animals per group in the experiments). The animals tested in the water maze, forced swimming, and latent inhibition were only used in one experiment. The rats tested in the DNMTP paradigm and in object recognition had previously been tested in other models (social interaction and basal locomotor activity), but had not received any other pharmacological treatment than the intrauterine MAM exposure.

\section{Compounds and Administration}

Methylazoxymethanol acetate (obtained from the National Cancer Institute Chemical Carcinogen Reference Standard Repository) was dissolved in saline. Vehicle solution was $0.9 \%$ saline. Compounds were given i.p. in a volume of $5 \mathrm{ml} / \mathrm{kg}$.

\section{Morris' Water Maze}

The rats were trained in Morris' water maze. The water maze consisted of a circular, black pool measuring $1.20 \mathrm{~m}$ in diameter $\times 0.45 \mathrm{~m}$ in height. The pool was filled with water $\left(21 \pm 1^{\circ} \mathrm{C}\right)$ to a depth of $27 \mathrm{~cm}$. A circular escape platform $(8 \mathrm{~cm}$ in diameter) was placed just below the water surface. A video camera monitored the behavior of the rats in the pool and the video signal was transmitted to a computer and analyzed using the Ethovision system from Noldus Information Technology. The test room contained several permanent extra maze cues such as the rat housing rack, laboratory table, posters on the walls, etc. The following two paradigms were performed.

Reference memory procedure. The platform remained in the same position (north) during the entire experiment. Before each trial, the rat was placed on the hidden platform for $15 \mathrm{~s}$ (intertrial period). The rats were subjected to four trials a day for four consecutive days. At the start of a trial, the rat was placed in the maze at one of the three different start positions at the perimeter of the maze (south, west, or east) facing the wall, and the first and last trials were always initiated from the south position. If the rat did not locate the platform within $60 \mathrm{~s}$, the rat was gently placed on the platform.

Reversal procedure. The platform was placed in a different quadrant every day (north $\rightarrow$ south $\rightarrow$ west $\rightarrow$ east) for 4 days. Before each trial, the rat was placed on the hidden platform for $15 \mathrm{~s}$ (intertrial period). The initial placement on the platform each day served to reveal the new location of the platform. The rats were subjected to four trials a day for 4 consecutive days. The rats were placed in the water maze from relative positions similar to those in the reference memory procedure (that is, opposite to the platform in the first and last trials each day). If the rat did not locate the platform within $60 \mathrm{~s}$, the rat was gently placed on the platform.

The following parameters were measured or calculated during each trial: path length $(\mathrm{m})$, escape latency $(\mathrm{s})$ to find the hidden platform, percent of path length near $(10 \mathrm{~cm})$ the sidewall (percent sidewall), number of animals not finding the platform within $60 \mathrm{~s}$ (nonfinders). 


\section{Delayed Non-Match-To-Position}

The animals were trained to perform the DNMTP paradigm in operant boxes (MED Associates Inc.). The rats were water deprived and water was used as a positive reinforcer. The rats had free access to water for $1 \mathrm{~h}$ a day $30 \mathrm{~min}$ after the daily session. The procedure requires the four training schedules shortly outlined below.

Panel. The light above the water panel was lit and the water (0.1 $\mathrm{ml}$ dipper) made available. After $5 \mathrm{~s}$ of drinking, the light was turned off and the water dipper removed. After $15 \mathrm{~s}$, the light was turned on again and the water made available. This continued for $20 \mathrm{~min}$. The animals were trained in this program only once.

Lever pressing. The rats learned to press a lever to initiate the delivery of water. Initially, water was presented. At this level, the rat only had the water available for $3 \mathrm{~s}$ after it entered the water panel. A lever placed next to the water panel was then introduced in the box (automatically). As the rat pressed the lever, the lever was retracted and the light above the water panel was turned on and water made available. After the rat collected the reinforcement, the procedure started over again, after an intertrial interval of $5 \mathrm{~s}$, by the introduction of the lever. The animals were trained to lever press for water for six sessions, each session lasting $20 \mathrm{~min}$ (except the first session, which lasted $60 \mathrm{~min})$.

Non-Match-To-Position. In this training schedule, the rats learned to perform the non-match-to-position (NMTP) paradigm. Initially, the rats were given a delivery of water as in 'lever pressing'. One of two levers placed opposite to the water panel, right or left (random), was then introduced into the box. When the rat pressed the lever (lever A), it was removed and the light at the water panel turned on and water made available. After the rat had had its water reinforcement, both levers were introduced in the box. If the rat pressed lever B (the lever opposite of the one initially pressed), both levers were removed and the light at the water panel was turned on and water made available (correct choice). Alternatively, at a press on lever A (same lever as the one initially pressed), the levers were removed and all light in the box was turned off for $2 \mathrm{~s}$ (error). After the trial follows an intertrial period lasting $5 \mathrm{~s}$ before a new trial is initiated with the introduction of either the left or the right lever. The animals were trained in this procedure for 40 sessions.

Delayed Non-Match-To-Position. The procedure was as in the 'NMTP' experiment, except that a delay $(0,5$, or $10 \mathrm{~s}$, random) was inserted after the initial press on the right or left lever before the reward was given. The animals were initially trained for 25 sessions in this procedure including the delay and the subsequent three sessions were used as the test sessions.

\section{Object Recognition}

The experiments were performed in a wooden box measuring $45 \times 65 \times 29 \mathrm{~cm}$. As objects we used either brown
1-1 glass bottles or transparent glass cylinders, and the known/new object was randomized for each rat. On the day before the test, rats were habituated to the test box for $10 \mathrm{~min}$. On the test day, rats were put in the test box, and after 3 min habituation two similar objects (brown glass bottles or transparent glass cylinders in a balanced design) were introduced in the two corners. The time spent investigating the two objects was scored in the next $3 \mathrm{~min}$ period (denoted pretest). The rats were then removed and put back in the housing cage. After a delay period of $20 \mathrm{~min}$, the rats were reintroduced to the test box and 3 min later the objects - one similar to that used in the pretest (known) and one the rat had never encountered before (new) - were introduced. The objects were placed in the same position as in the pretest. In the next 3 min, the time spent investigating each of the objects was scored. All scoring was performed using the Observer ${ }^{\circledR}$ (Noldus Information Technology). The test box was cleaned with soap between each trial; the objects were always handled using rubber gloves and were washed with water and soap between trials.

\section{Forced Swimming}

The rats were put into a container $45 \mathrm{~cm}$ high and $19 \mathrm{~cm}$ in diameter, filled with water $\left(23^{\circ} \mathrm{C}\right)$ up to a depth of $20 \mathrm{~cm}$. The animals were left to swim in the water for $15 \mathrm{~min}$ (test), and then dried and put back in their cages. After $24 \mathrm{~h}$, the procedure was repeated for $5 \mathrm{~min}$ (re-test). The experiment (both days) was recorded on a videotape for later manual analysis of escape-oriented behavior. The following behaviors were interpreted as escape-oriented behaviors: swimming, climbing, and diving. The data were analyzed in 5 min time bins.

\section{Latent Inhibition}

The experiment was performed using an automated shuttle box $(42 \times 16 \times 20 \mathrm{~cm}) \quad($ ENV-010M, MED-Associates) divided into two compartments by a partition with one opening. On the first day, the animals were left undisturbed in the shuttle box for $50 \mathrm{~min}$ with the houselights turned on. On the second day, the animals were divided into groups of pre-exposed (PE) and non-pre-exposed (NPE). The PE rats were subjected to 50 presentations (10-60 s randomized intertrial interval) of the to-be-conditioned stimulus (cuelight on for $10 \mathrm{~s}$ ). The NPE rats were left undisturbed in the test box with only the houselights on for a corresponding duration. On the third day (test session), the animals were subjected to 100 trials of avoidance learning (10-60 s randomized intertrial interval). Upon presentation of a light signal, the rat had $10 \mathrm{~s}$ to avoid getting a $0.5 \mathrm{~mA}$ scrambled foot shock by moving to the other compartment (avoidance). If the rat did not move to the other compartment within the $10 \mathrm{~s}$, a foot shock of a maximum duration of $10 \mathrm{~s}$ was delivered. The shock was terminated if the rat moved to the other compartment. The position of the animal and crossings from one compartment to the other were detected by two photocells placed on either side of the dividing wall. The outcome measure was the number of avoidances (ie rats moving to the other compartment of the shuttle box after the presentation of the light, but before the shock was delivered). 


\section{Statistics}

Morris' water maze. The effects of the prenatal MAM treatment on path length $(\mathrm{m})$, escape latency $(\mathrm{s})$, percent sidewall, and speed $(\mathrm{m} / \mathrm{s})$ were analyzed using two-way repeated measures (RM) ANOVA with days as RM. The latency data were also expressed as percent of 'nonfinders', that is, percent of trials that lasted $60 \mathrm{~s}$, and analyzed using a nonparametric $\chi^{2}$ test (Siegel, 1956) on each day.

Non-match-to-position. The number of animals learning to press the lever during the 'lever-pressing' procedure did not differ (14 controls and 14 MAM-treated). Additional rats from each group (two controls and one MAM-treated) started to lever press during the NMTP procedure and were included in the DNMTP (the data from these three animals were not included in the NMTP statistics, but only in the DNMTP statistics). The NMTP phase of the training was evaluated by two-way RM ANOVA (session as the repeated factor). The DNMTP was evaluated after an initial training phase consisting of 25 sessions. Three consecutive sessions were then evaluated using three-way RM ANOVA (with session as the repeated factor).

Object recognition. The difference in investigation time, between controls and MAM-treated rats, on the pretest was evaluated by Student's $t$-test. The investigation time during the test was evaluated by two-way RM ANOVA (known/new object as one factor and control/MAM as the other). Post hoc examination was carried out by a Tukey test.

Forced swim. The escape-oriented behaviors displayed by the rats on day 1 were evaluated by two-way RM ANOVA (with time bin being the repeated factor). The difference between control and MAM-treated rats in the 5 min test on day 2 was evaluated by a $t$-test.

Latent inhibition. The number of avoidances during the test session was evaluated by two-way ANOVA (control/ MAM as one factor and PE/NPE as the other).

\section{RESULTS}

\section{Morris Water Maze}

In the experiment using the 'reference memory' paradigm, there was no difference between the MAM-treated rats and the controls on any of the parameters measured (Figure 1). The two-way ANOVA with day as RM revealed an effect of test day on all parameters (distance $\mathrm{F}(3,42)=41.9$, latency $\mathrm{F}(3,42)=40.4$, and percent sidewall $\mathrm{F}(3,42)=38.6$; $P<0.001$ ), indicating the acquisition of the task.

In the reversal procedure (Figure 2), the analysis also revealed an effect of test day on all parameters (distance $\mathrm{F}(3,42)=12.2$, latency $\mathrm{F}(3,42)=13.4$, and percent sidewall $\mathrm{F}(3,42)=20.4 ; P<0.001)$. In addition, there was an interaction between group (MAM/CON) and test day. This interaction was evident on all measures (distance $\mathrm{F}(3,42)=2.90$, latency $\mathrm{F}(3,42)=2,89$, and percent sidewall $\mathrm{F}(3,42)=2.90 ; P=0.046$; same effect on all measures); however, the post hoc comparison only found significant difference between the groups on the percent sidewall parameter (day 3, $q=3.32, P=0.025$ ). The analysis of the percent nonfinders revealed significant differences between the groups on days 2 and 3 of testing (day $2, \chi^{2}=4.79,1 \mathrm{df}$, $P=0.029$; day $\left.3, \chi^{2}=4.26,1 \mathrm{df}, P=0.04\right)$. Thus, it appears that there was no difference between the groups on day 1 , which is in agreement with the reference paradigm, and a difference between the groups was evident on days 2 and 3 of testing, and disappeared on day 4, possibly because both groups reached a plateau.

There was no difference between the control and the MAM-treated groups on the speed of swimming in either of the versions of the test.

\section{Delayed Non-Match-To-Position}

The analysis of the NTMP phase of the experiment (Figure 3a) showed a clear effect of the session $(\mathrm{F}(39,1014)=21.5, P<0.001)$ and an interaction between the session and group $(\mathrm{F}(39,1014)=1.60, P=0.012)$, and the post hoc analysis revealed a difference between the
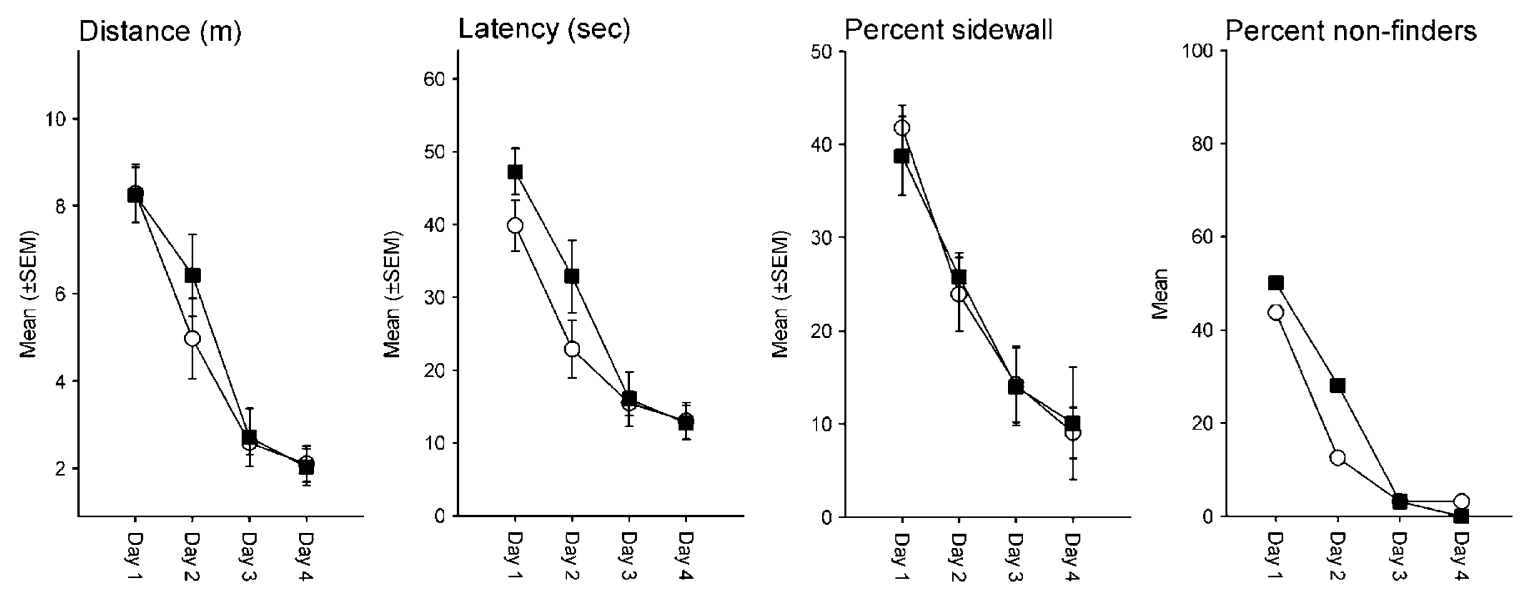

Figure I Effect of MAM vs saline treatment on GDI7 on performance in the reference memory paradigm of the Morris' water maze. The data presented are the mean of four consecutive trials. There was no significant difference between the two groups on any measure. Each treatment group included eight rats. Open circles represent controls and filled squares represent MAM. 

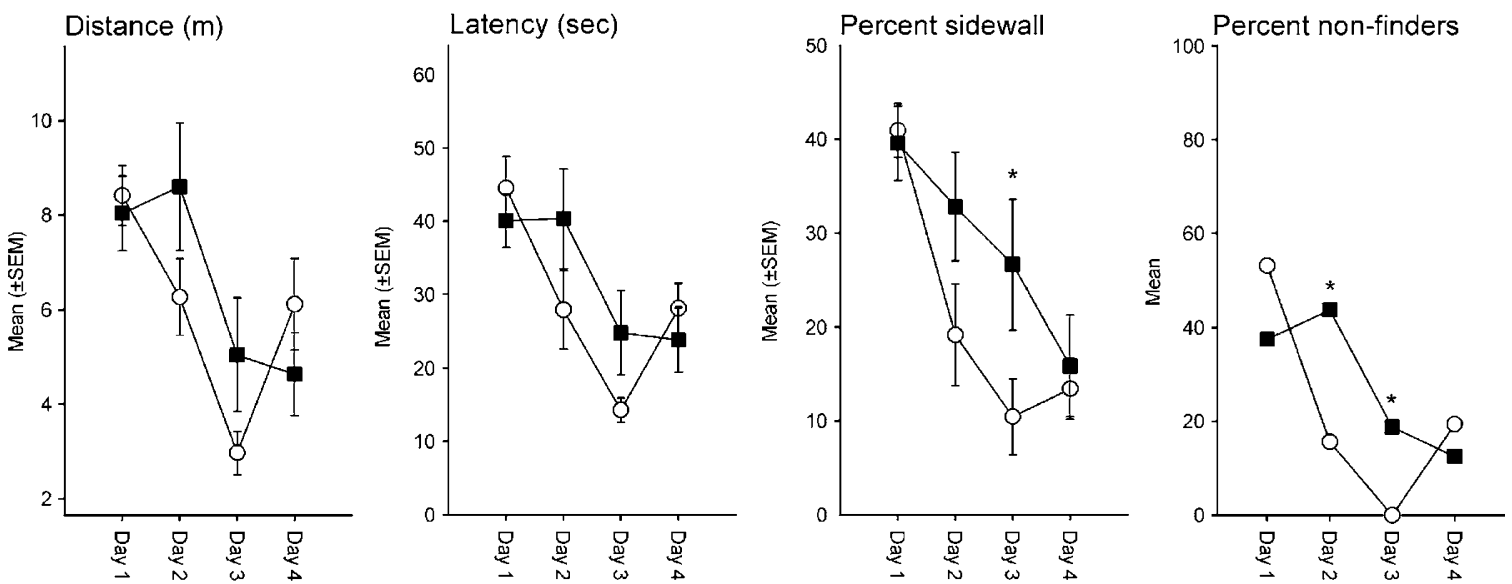

Figure 2 Effect of MAM vs saline treatment on GDI7 on performance in the reversal learning paradigm of the Morris' water maze. The data presented are the mean of four consecutive trials. There was an overall significance interaction between group and day on all measures (distance, latency, and percent sidewall). Furthermore, there were significantly more nonfinders in the MAM group than in the controls on days 2 and 3. *Significance $(P<0.05)$ according to the Tukey post hoc test (or the $\chi^{2}$ test). Each treatment group included eight rats. Open circles represent controls and filled squares represent MAM.

groups at session nos. 17, 21, 23, and $28(P<0.05)$. Although a numerical difference is noted in favor of the MAM rats on the first nine sessions, none of these reached statistical significance. There were no group effects in the analysis of the three consecutive DNMTP sessions $(\mathrm{F}(1,29)=0.07$, $P=0.80$; Figure $3 \mathrm{~b}$ ) and also no group differences during the 25 training sessions (data not shown). The analysis revealed a trend toward an effect of the day $(F(2,58)=3.00$, $P=0.058)$. There was a strong effect of delay period $(\mathrm{F}(2,58)=17.221, P<0.001)$ showing that the performances of the rats are delay-dependent.

\section{Object Recognition}

The MAM-treated rats spent significantly less time exploring the objects on the pretest $(t(12)=2.60, P=0.023$; see Figure 4). There was also a main effect of the group in the test session $(\mathrm{F}(1,12)=17.0, P=0.001)$, indicating that the MAM-treated rats spent less time exploring the objects. There was no main effect of the known/new object factor; however, there was an interaction between the group and the known/new factor $(F(1,12)=7.53, P=0.018)$. This interaction was caused by a significant difference between the known/new object in controls $(q=4.64, P=0.007)$, but not in the MAM-treated rats $(q=0.84, P=0.562)$. Furthermore, there was no difference between the amounts of time the animals in each group spent exploring the known object $(q=1.73, P=0.234)$, whereas the controls spent a longer time exploring the new object than the MAM-treated did $(q=6.93, P<0.001)$.

\section{Forced Swimming}

The results of the forced swimming experiment are shown in Figure 5. The two-way ANOVA of the data from day 1 (with the $5 \mathrm{~min}$ time bin as the repeated factor) showed that there were main effects only of time $(F(2,24)=31,5$, $P<0.001)$. There was no significant interaction between treatment (MAM vs control) and time. The Tukey post hoc test showed that the rats showed less escape-related behaviors in the $5-10$ and $10-15$ min periods than in the initial $5 \mathrm{~min}(P<0.001)$. The MAM rats spent significantly more time than the controls on escape-related behaviors on day $2(t(12)=2.58, P=0.024)$. These data indicate that the behaviors of the control rats were affected by the swimming experience on the previous day, whereas this was not the case in the MAM-treated rats.

\section{Latent Inhibition}

The latent inhibition experiment revealed an interaction $(\mathrm{F}(1,32)=4.42, P=0.044)$ between the treatment group and PE/NPE (Figure 6). The Tukey post hoc test revealed that this was evident as a significant difference between $\mathrm{PE}$ and NPE in the controls $(\mathrm{q}=4.67, P=0.003)$, but not in the MAM-treated rats $(q=0.46, P=0.747)$. The experiment further showed that there was an effect of treatment group (CON/MAM) on the number of avoidances made by the NPE rats $(q=2.93, P=0.047)$, suggesting a learning deficit in the MAM rats.

\section{DISCUSSION}

The present experiments demonstrate that gestational disruption of neurogenesis on GD17 disrupts cognitive performance in a number of different paradigms. As the MAM treatment decreases the size of various brain structures, such as the hippocampus, cortex, and thalamus (Moore and Grace, 1997), it could be expected that the deficits in cognition would be rather extensive. The deficits, however, seem to be limited to only some cognitive skills, whereas others are left undisturbed. The MAM-treated rats had no deficits in learning and remembering the position of the hidden platform in the reference memory version of the Morris water maze. In a preliminary abstract from our laboratory (Didriksen et al, 1999), it was described that MAM-treated rats had a deficit in this task compared to controls; however, the experiment has now been repeated several times and no deficits have been observed. The 


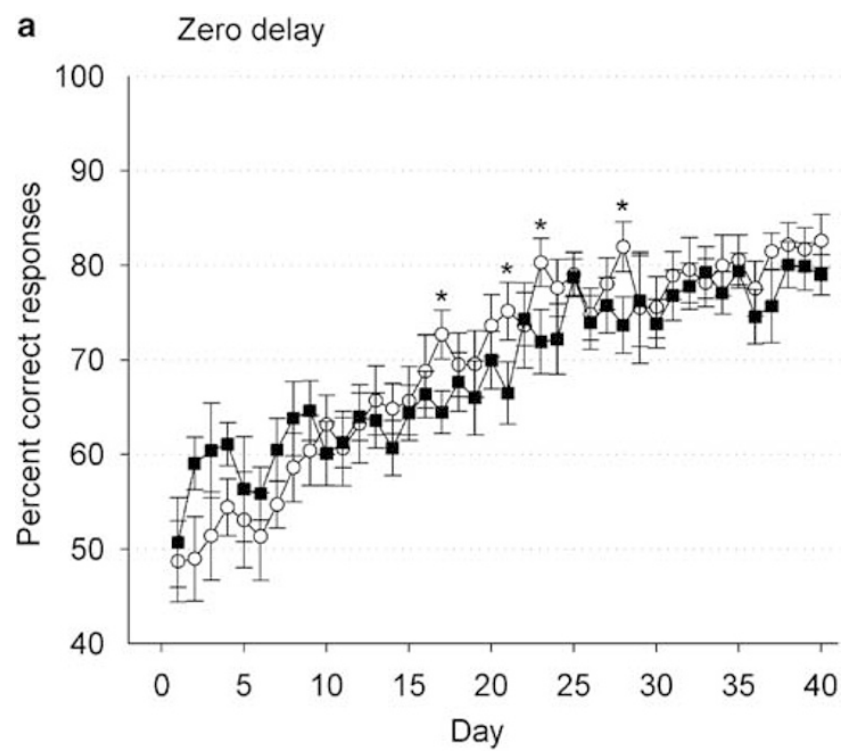

b Delay 0-10

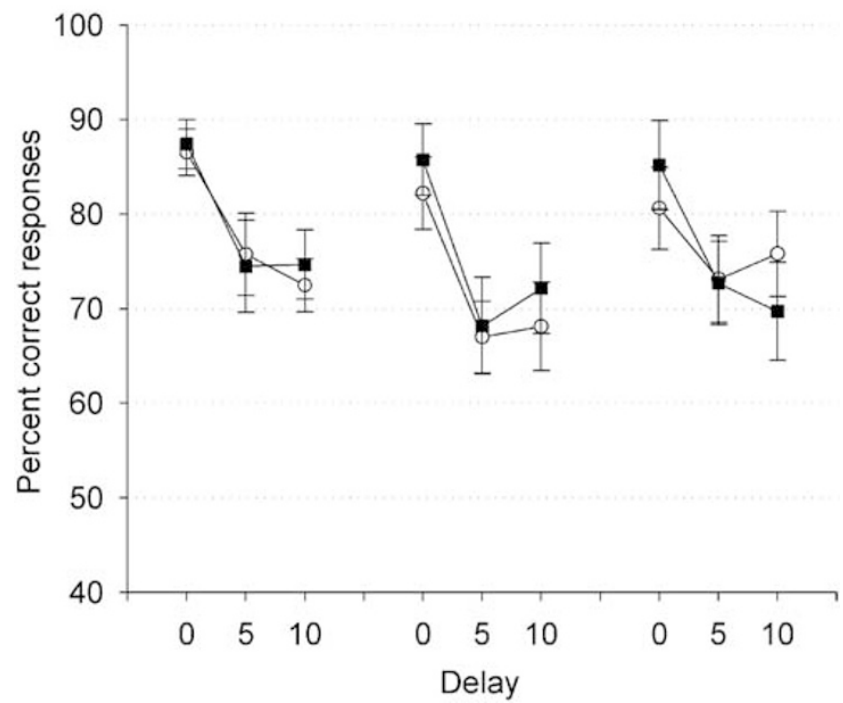

Figure 3 (a) The mean percent correct responses ( \pm SEM) of the groups during the 40 days of acquisition of the NMTP paradigm. There was no overall effect of the group $(n=\mid 4)$; however, an interaction between group and day revealed differences at four time points. *Significance $P<0.05$ according to a Tukey post hoc test. (b) The mean percent correct responses $( \pm$ SEM $)$ during three consecutive days of DNMTP. There was no significant difference between the groups (CON, $n=16$; MAM, $n=15)$. Open circles represent controls and filled squares represent MAM.

discrepancy between the initial preliminary result and the repetitions is unknown. The reference memory task has been proven to be sensitive to hippocampal lesions (Morris, 1984; Morris et al, 1982). However, the lesions have to exceed $10 \%$ of the hippocampal volume to affect spatial learning, and in addition the dorsal rather than the ventral hippocampus supports spatial learning (Moser et al, 1993). Even though the MAM treatment is given at the time of the peak proliferation of the hippocampus (Bayer and Altman, 1995) and thus causes maldevelopment of the structure (Moore and Grace, 1997; Flagstad et al, 2004), this is not associated with deficits in spatial learning.
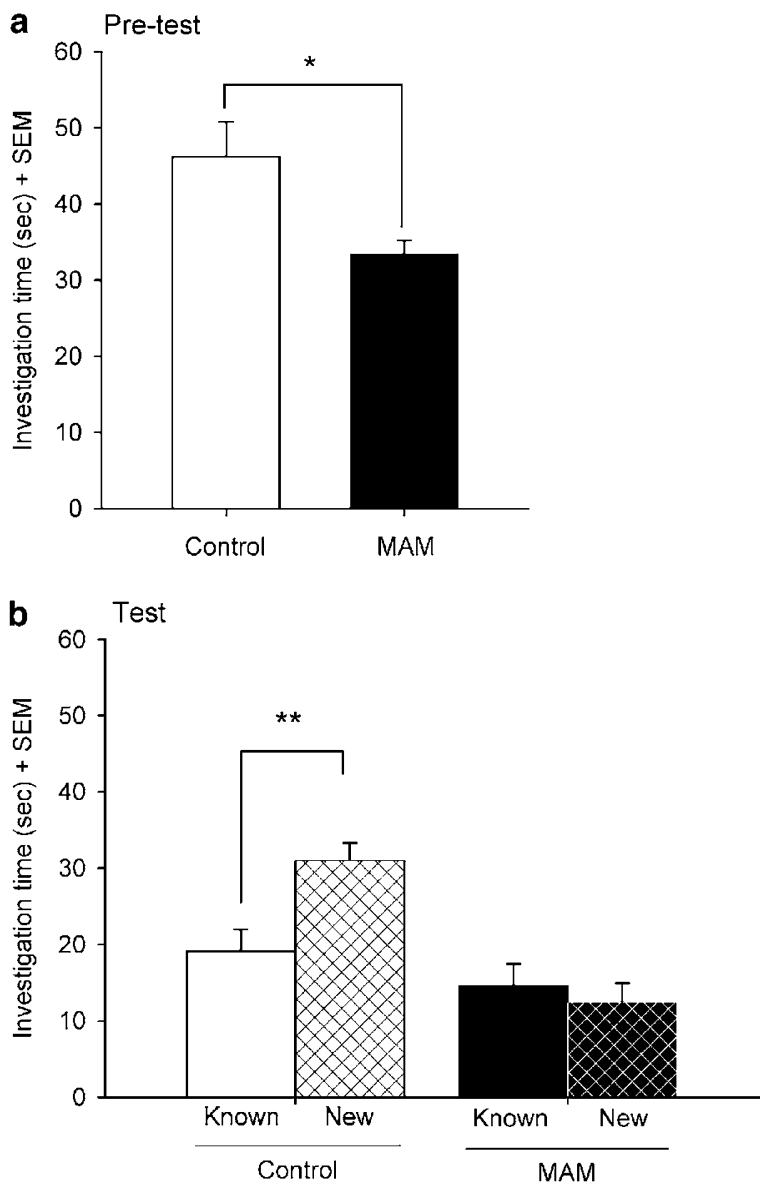

Figure 4 (a) Mean investigation time $( \pm S E M)$ during the pretest of the object recognition paradigm. The MAM-treated rats (black bar) spent significantly less time than the controls (white bar) investigating the objects. $* P<0.05$, t-test. (b) Mean investigation time $( \pm$ SEM) of the known (solid bars) and the new (hatched bars) object during the object recognition test. The control rats (white bars) spent significantly more time on the new vs the known object, whereas the MAM-treated rats (black bars) did not. $n=7$ for both groups. ${ }^{*} * P<0.0$ I, ${ }^{*} * * P<0.001$ according to a Tukey post hoc test.

In the reversal paradigm of the water maze task, the MAM-treated rats performed significantly worse than the controls on days 2 and 3 of testing. On the last day, however, they performed as well as the controls. Thus, it does not appear that the MAM treatment interferes with spatial working memory, as this should also result in a deficit on the last day of testing. The MAM treatment seems, however, to affect the search strategy of the rats. When the platform is moved, the rats spend more time swimming along the sidewall, rather than searching in the central part of the maze where the platform is positioned. Slower acquisition during reversal training has previously been described in medial prefrontal cortex-lesioned rats (de Bruin et al, 1994; Lacroix et al, 2002) and these rats had, as in the present experiment, no deficit in the reference task.

The slower acquisition of a reversal-learning task in medial prefrontal-lesioned rats has been interpreted as a deficit in attentional processes or lack of behavioral flexibility (de Bruin et al, 1994; Lacroix et al, 2002). Attentional processes depend among others on an anterior neuronal network involving dorsolateral prefrontal and 


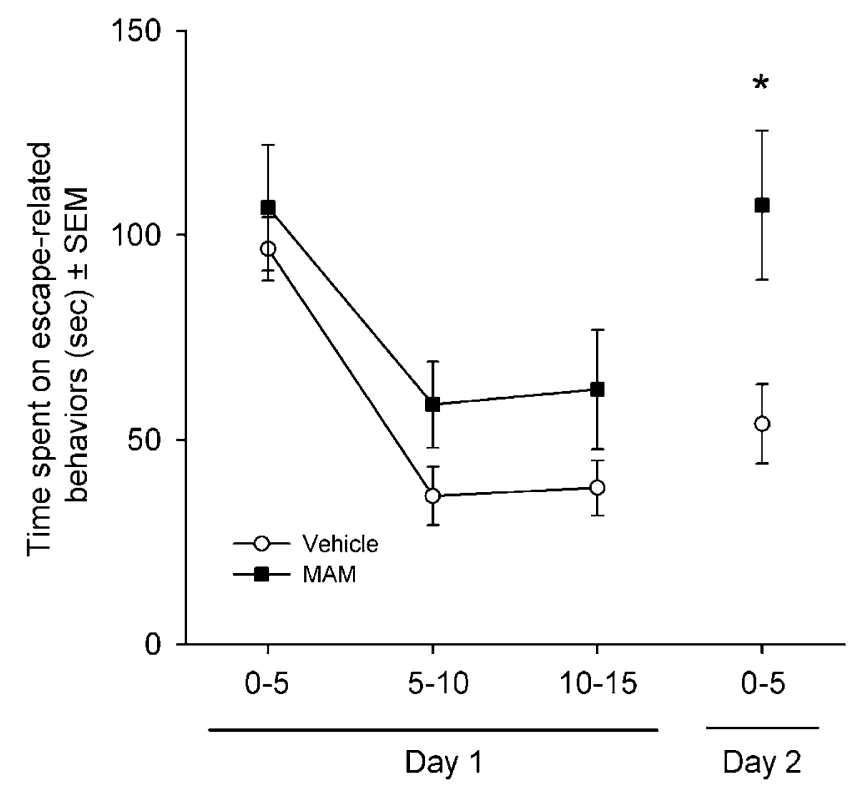

Figure 5 Mean time spent on escape-related behaviors $( \pm S E M)$ during the three 5 min time bins on day I and the one 5 min time bin on day 2 of the forced swimming test. The MAM group (filled squares) spent significantly more time on escape-related behaviors than the controls (open circles), $P=0.044$, two-way RM ANOVA, $n=7$ for both groups.

anterior cingulated cortex. This network is believed to be involved in a top-down control of selective attention and executive functions (Arrington and Carr, 2003). The disturbed search strategy in the reversal paradigm could suggest that the developmental disturbance induced by the MAM GD17 treatment interferes with attentional processes and spontaneous flexibility/planning efficiency, which is a component of executive function, that is, relies on frontal and anterior cingulated cortex, whereas the hippocampal dysgenesis induced by the MAM treatment is not gross enough to cause a deficit in the reference memory version of the test. This result shows good homology to schizophrenia where selective attention as well as executive functions measured as planning efficiency is impaired in drug-naïve first-episode schizophrenic patients (Fagerlund et al, 2004). Other abnormalities, such as visual or motor abnormalities, or increased anxiety or stress could also affect behavior; however, this is most likely not the case, as the groups performed comparably in the reference memory procedure in the water maze.

Another cognitive function, which is associated with the prefrontal cortical function, is working memory. Working memory may be defined as the capacity to hold information online over short delays while that information is integrated with other ongoing mental operations. A number of studies have shown that schizophrenic patients have deficient working memory (Goldberg et al, 1987; Gold et al, 1994, 1997), and imaging studies have confirmed that this is associated with abnormal activation of the prefrontal cortex (Manoach, 2003).

The present investigation of working memory using the NMTP paradigm showed no consistent difference between the performances of the MAM-treated rats and the controls, either in the nondelay condition or in the delay condition. a
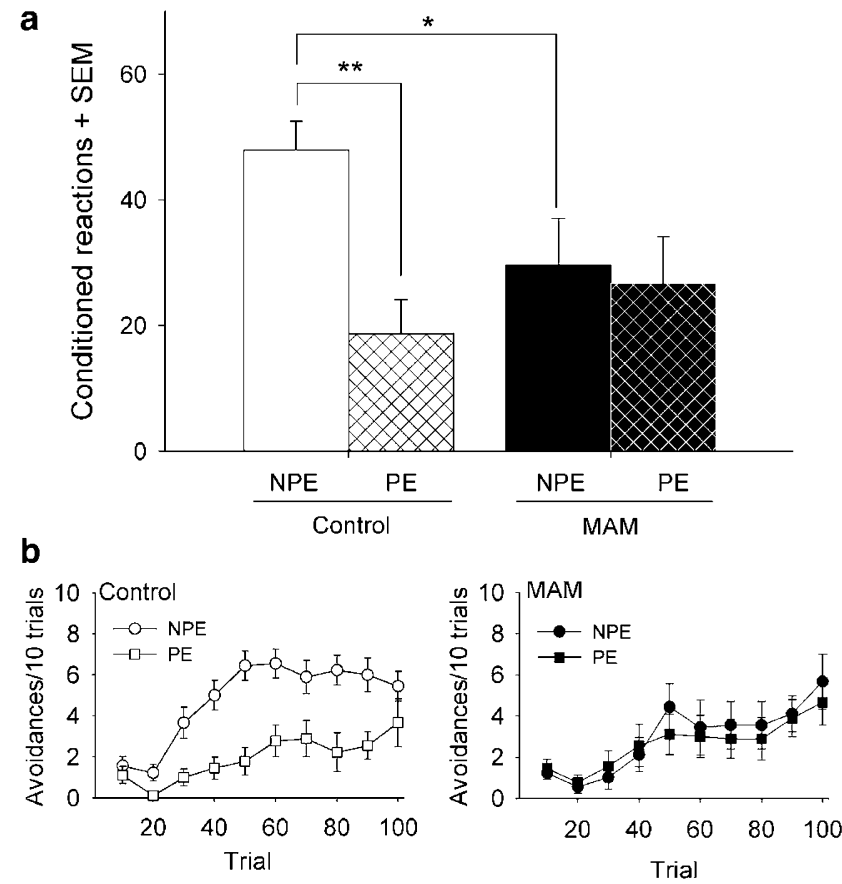

Figure 6 (a) Number of avoidance responses (+ SEM) during a 100 trial learning session in a two-way avoidance shuttle box. The graph shows the performance of control (white bars) and MAM-treated (black bars) either non-pre-exposed (NPE, solid bars) or pre-exposed (PE, hatched bars) to the to-be-conditioned stimuli; $n=9$ for all groups. ${ }^{*} P<0.05$, ** $P<0.0$ I according to a Tukey post hoc test. (b) The number of avoidances made during the conditioning in 10 trial bins. Top graphs are the controls and bottom graphs the MAM-treated.

The controls were superior only in four sessions out of the 40 during the learning of the NMTP paradigm, and this did not appear to be a consistent effect. In the DNMTP paradigm, a delay-dependent decline was apparent, but no differences between groups were observed. The accuracy of the performance in the task is believed to be dependent on prefrontal cortical integrity, as lesions decrease performance in the task, although the published data suggest that the deficits of the prefrontal cortex-lesioned rats are apparent only in the beginning of training (van Haaren et al, 1985, 1988). In the present experiment, we first trained the animals on zero delay and then after extensive training switched them to the delay condition; this could possibly explain the lack of effect of the MAM treatment. Also, it is possible that under the present protocol the animals use a movement-mediated strategy: that the choice of the rat after the delay is mediated from the body position of the animal rather than by information kept online in working memory. Furthermore, during development, passively holding information available in working memory as well as recognition and recall from long-term memory is developed before active use and manipulations of information (executive functions), and as such not as dependent on later maturation of the prefrontal cortex (Diamond, 2002). This might suggest that the disturbances in prefrontal functions in the MAM rats in the present study are related to a later maturation of this structure, and hence less radical than a dysfunction of all processes governed by the prefrontal cortex. 
The conclusion of the experiment is, however, that the MAM-treated rats under the present conditions show no sign of a working memory dysfunction in keeping with the water maze data and thus that the model does not show face validity as regard to patients within the schizophrenic spectrum demonstrating this deficit.

The object recognition model is a spontaneous recognition test based on the natural bias of rats toward exploring novel objects (Ennaceur and Delacour, 1988). If a rat, as observed for the MAM-treated rats in the present experiment, does not preferentially explore the 'new' object on the retention test, it is inferred that the rat does not remember the 'known' object. Thus, the MAM-treated rats are thought to have a deficit in short-term object recognition memory.

Neuroimaging studies in human healthy volunteers during object recognition have shown activation of brain regions implicated in schizophrenia, that is, the medial temporal lobes, prefrontal areas, and the thalamus (Schacter et al, 1995, 1997). As would be expected from this, schizophrenic patients demonstrate impaired performance in the recognition of visually presented objects (Aleman et al, 1999; Heckers et al, 2000). Furthermore, this deficit is associated with abnormal activation of at least the prefrontal cortex and the thalamus (Heckers et al, 2000).

The interpretation of the present results should be treated with caution as other factors can influence the exploration. As the method is based on the rats' natural bias toward exploring novel objects, factors other than mnemonic capacities, such as lack of general exploratory drive and/ or the ability to initiate actions (which relate closer to the negative symptoms of schizophrenia than to the cognitive deficits), could affect the investigation of the novel, as compared to the known, object. We cannot exclude this possibility in the present experiment as the MAM-treated animals spend less time exploring the objects both in the pretest and the test. Consequently, the difference between MAM and controls could be the result of a deficit in shortterm object recognition memory and/or a lack of exploratory drive or ability to initiate actions. Both these explanations would be highly relevant to schizophrenia, but relating to different clinical symptoms, that is, a cognitive deficit $v s$ part of the negative symptomatology.

In the forced swimming paradigm (Porsolt et al, 1978), the animals swim around attempting to escape, which is impossible, and eventually assume an immobile posture called 'floating'. On a subsequent test, the animals more readily adopt the 'floating' behavior, indicating that the animals have learned that they cannot escape. Originally, this behavioral response was called 'behavioral despair'; the immobility might reflect a successful strategy, which conserves energy and allows the animal to float for prolonged periods of time, thereby improving its chances for survival (West, 1990). The MAM-treated animals showed more escape-related behaviors in the test (day 2). The same exaggerated response has been reported in other developmental models of schizophrenia like the neonatal hippocampal lesion or the neonatal amygdala lesion model (Daenen et al, 2001). In normal rats, the forced swim paradigm increases extracellular dopamine in the prefrontal cortex, but not in the nucleus accumbens (Tucci et al, 1994). In addition, the activation of the prefrontal cortex during the task has been established by 2-deoxyglucose experi- ments (Duncan et al, 1993). If dopamine response in the prefrontal cortex is inhibited (for example, by 6-OHDAinduced dopamine depletion), the animal does not adapt to the situation, but keeps swimming/struggling (Ravard et al, 1990; Espejo and Minano 1999). This could be interpreted as perseveration and underlines the importance of the prefrontal cortex in the adaptive response in a stressful situation. Abnormalities in both prefrontal dopaminergic sensitivity (Grace et al, 1998) and prefrontal modulation of subcortical dopaminergic activity (Flagstad et al, 2004) have been reported in MAM GD17-exposed rats; thus, it could be hypothesized that the aberrant behavior displayed by the MAM-treated rats could be caused by a prefrontal deficit. The MAM rats might show increased escape-related behavior because they find the situation more stressful or anxiogenic; this would be well in line with the evidence of an abnormal drive of the amygdala in this animal model (Grace and Moore, 1998).

In the latent inhibition experiment, control rats preexposed to the conditioning cue showed pronounced latent inhibition, whereas the MAM-treated showed none. The latent inhibition experiment, however, also revealed that under the present experimental setup, the MAM-treated rats had a slower acquisition of the conditioned avoidance. The deficit noted in this paradigm appears to be selective for rats exposed to MAM late during gestation, as it has recently been reported that exposure to MAM at embryonic days 915 does not affect latent inhibition (or two-way avoidance learning) in a similar two-way shuttle box procedure (Jongen-Rêlo et al, 2004).

It has been demonstrated using microdialysis during avoidance learning in a shuttle box that establishment of an avoidance behavior in gerbils correlates with transient high dopamine levels in the medial prefrontal cortex (Stark et al, 2000, 2001, 2004). This has been assumed to reflect the involvement of prefrontal dopamine in the working memory processes needed for the signal evaluation and formation of the behavioral response (Stark et al, 2004). Impaired dopamine transmission in the prefrontal cortex has been demonstrated previously in rats treated with MAM at GD17, and this could be a possible explanation for the impaired acquisition of the conditioned avoidance in this study.

Deficits in conditional associative learning have been described in schizophrenic patients. This deficit has in one study been shown to be selective for a visual warning signal of an aversive event compared to a nonaversive event (Kosmidis et al, 1999). Kosmidis et al (1999) hypothesized that the increased arousal by the aversive event interfered with the ability to use feedback constructively and modify their behavior appropriately. This selective deficit is also observed in our studies where no deficit was noted in the acquisition of the lever-pressing task in the NMTP paradigm, but a deficit was noted in avoidance learning. Another finding of the present paper, namely the increased escape-related behavior displayed by the MAM-treated rats in the second session of the forced swim test, could equally be caused by increased arousal interfering with the ability to use feedback to modify behavior.

The MAM-treated rats did show acquisition (although not optimal) and it should consequently be possible to demonstrate latent inhibition; however, we cannot totally 
dismiss that the slower acquisition might bias the latent inhibition assessment.

Disruption of latent inhibition is characteristic of the acute state of exacerbation of positive symptoms and is believed to be caused by the exaggerated subcortical dopamine activity (Gray et al, 1995, 1997). Increased subcortical dopamine activity in the MAM-treated rats has been demonstrated in an amphetamine challenge microdialysis study and is further indicated by increased locomotor activity when tested in the open field both at mild stress and upon amphetamine challenge (Flagstad et al, 2004). Thus, it is plausible that with the aversive conditioning employed in the latent inhibition experiment (foot shock), the difference between the MAM-treated rats and controls was caused by different subcortical dopaminergic activities.

Altogether, in addition to the behavioral changes relevant for the positive and negative symptoms of schizophrenia, rats exposed to MAM at GD17 display some cognitive deficits that may be of relevance to schizophrenia. Thus, the present model could be used to investigate novel treatment regimes aiming to treat some of the cognitive disturbances of schizophrenia.

\section{REFERENCES}

Aleman A, Hijman R, de Haan EH, Kahn RS (1999). Memory impairment in schizophrenia: a meta-analysis. Am J Psychiatry 156: $1358-1366$.

Andreasen NC (1999). A unitary model of schizophrenia. Arch Gen Psychiatry 56: 781-787.

Andreasen NC, Paradiso S, O'Leary DS (1998). 'Cognitive dysmetria' as an integrative theory of schizophrenia: a dysfunction in cortical-subcortical-cerebellar circuitry? Schizophr Bull 24: 203-218.

Andreasen NC, Rezai K, Alliger R, Swayze VW, Flaum M, Kirchner $\mathrm{P}$ et al (1992). Hypofrontality in neuroleptic-naive patients and in patients with chronic schizophrenia. Assessment with xenon 133 single-photon emission computed tomography and the Tower of London. Arch Gen Psychiatry 49: 943-958.

Arnold SE, Hyman BT, Van-Hoesen GW, Damasio AR (1991). Some cytoarchitectural abnormalities of the entorhinal cortex in schizophrenia. Arch Gen Psychiatry 48: 625-632.

Arrington C, Carr T (2003). Attention. In: Schiffer R, Rao S, Fogel B (eds). Neuropsychiatry, 2nd edn. Lippincott: Philadelphia. pp 405-426.

Balduini W, Lombardelli G, Peruzzi G, Cattabeni F (1991). Treatment with methylazoxymethanol at different gestational days: physical, reflex development and spontaneous activity in the offspring. Neurotoxicology 12: 179-188.

Bayer S, Altman J (1995). Neurogenesis and neuronal migration. In: Paxinos G (ed). The Rat Nervous System, 2nd edn. Academic Press: San Diego, CA. pp 1041-1078.

Berman KF, Torrey EF, Daniel DG, Weinberger DR (1992). Regional cerebral blood flow in monozygotic twins discordant and concordant for schizophrenia. Arch Gen Psychiatry 49: 927-934.

Buchsbaum MS, Haier RJ, Potkin SG, Nuechterlein K, Bracha HS, Katz $M$ et al (1992). Frontostriatal disorder of cerebral metabolism in never-medicated schizophrenics. Arch Gen Psychiatry 49: 935-942.

Buka SL, Tsuang MT, Torrey EF, Klebanoff MA, Bernstein D, Yolken RH (2001). Maternal infections and subsequent psychosis among offspring. Arch Gen Psychiatry 58: 1032-1037.
Conrad AJ, Abebe T, Austin R, Forsythe S, Scheibel AB (1991). Hippocampal pyramidal cell disarray in schizophrenia as a bilateral phenomenon. Arch Gen Psychiatry 48: 413-417.

Daenen EW, Van der Heyden JA, Kruse CG, Wolterink G, Van Ree JM (2001). Adaptation and habituation to an open field and responses to various stressful events in animals with neonatal lesions in the amygdala or ventral hippocampus. Brain Res 918: 153-165.

Daenen EW, Wolterink G, Gerrits MA, Van Ree JM (2002). The effects of neonatal lesions in the amygdala or ventral hippocampus on social behaviour later in life. Behav Brain Res 136: 571-582.

de Bruin JP, Sanchez-Santed F, Heinsbroek RP, Donker A, Postmes $P$ (1994). A behavioural analysis of rats with damage to the medial prefrontal cortex using the Morris water maze: evidence for behavioural flexibility, but not for impaired spatial navigation. Brain Res 652: 323-333.

Diamond A (2002). Normal development of prefrontal cortex from birth to young adulthood: cognitive functions, anatomy, and biochemistry. In: Stuss D, Knight RT (eds). Principles of Frontal Lobe Functions. Oxford University Press: Oxford. pp 466-503.

Didriksen M, Sams-Dodd F, Arnt J (1999). Cognitive deficits in rats treated prenatally with the mitosis inhibitor methylazoxymethanol (MAM) at different time points during gestation. Schizophr Res 36: 164.

Duncan GE, Johnson KB, Breese GR (1993). Topographic patterns of brain activity in response to swim stress: assessment by 2-deoxyglucose uptake and expression of Fos-like immunoreactivity. J Neurosci 13: 3932-3943.

Ennaceur A, Delacour J (1988). A new one-trial test for neurobiological studies of memory in rats. 1: Behavioral data. Behav Brain Res 31: 47-59.

Espejo EF, Minano FJ (1999). Prefrontocortical dopamine depletion induces antidepressant-like effects in rats and alters the profile of desipramine during Porsolt's test. Neuroscience 88: 609-615.

Fagerlund B, Mackeprang T, Gade A, Glenthoj BY (2004). Effects of low-dose risperidone and low-dose zuclopenthixol on cognitive functions in first-episode drug-naive schizophrenic patients. CNS Spectr 9: 364-374.

Flagstad P, Mørk A, Glenth $\varnothing$ j BY, van Beek J, Michael-Titus AT, Didriksen M (2004). Disruption of neurogenesis at gestational day 17 in the rat causes behavioral changes relevant to positive and negative schizophrenia symptoms and alters amphetamineinduced dopamine release in nucleus accumbens. Neuropsychopharmacology 29: 2052-2064.

Fujii DE, Wylie AM (2003). Neurocognition and community outcome in schizophrenia: long-term predictive validity. Schizophr Res 59: 219-223.

Fujii DE, Wylie AM, Nathan JH (2004). Neurocognition and longterm prediction of quality of life in outpatients with severe and persistent mental illness. Schizophr Res 69: 67-73.

Gold JM, Carpenter C, Randolph C, Goldberg TE, Weinberger DR (1997). Auditory working memory and Wisconsin Card Sorting Test performance in schizophrenia. Arch Gen Psychiatry 54: $159-165$.

Gold JM, Hermann BP, Randolph C, Wyler AR, Goldberg TE, Weinberger DR (1994). Schizophrenia and temporal lobe epilepsy. A neuropsychological analysis. Arch Gen Psychiatry 51: 265-272.

Goldberg T, Gold J (1995). Neurocognitive deficits in schizophrenia. In: Hirsch SR, Weinberger DR (eds). Schizophrenia, 1st edn. Blackwell Science Ltd: Berlin. pp 146-162.

Goldberg TE, Weinberger DR, Berman KF, Pliskin $\mathrm{NH}$, Podd MH (1987). Further evidence for dementia of the prefrontal type in schizophrenia? A controlled study of teaching the Wisconsin Card Sorting Test. Arch Gen Psychiatry 44: 1008-1014. 
Gourevitch R, Rocher C, Pen GL, Krebs MO, Jay TM (2004). Working memory deficits in adult rats after prenatal disruption of neurogenesis. Behav Pharmacol 15: 287-292.

Grace AA (2000). Gating of information flow within the limbic system and the pathophysiology of schizophrenia. Brain Res Rev 31: $330-341$.

Grace AA (2003). Gating within limbic-cortical circuits and its alteration in a developmental disruption model of schizophrenia. Clin Neurosci Res 3: 333-338.

Grace AA, Moore H (1998). Regulation of information flow in the nucleus accumbens: a model for the pathophysiology of schizophrenia. In: Lenzenweger M, Dworkin R (eds). Origins and Development of Schizophrenia: Advances in Experimental Psychopharmacology. American Psychiatric Press: Washington, DC. pp 123-160.

Grace AA, Moore H, Lavin A (1998). Disruption of temporal cortical development as an animal model of schizophrenia: alterations in prefrontal cortical-limbic interactions. Int $J$ Neuropsychopharmacol 1: S24.

Grawe RW, Levander S (2001). Neuropsychological impairments in patients with schizophrenia: stability and prediction of outcome. Acta Psychiatr Scand Suppl 408: 60-64.

Gray JA, Joseph MH, Hemsley DR, Young AM, Warburton EC, Boulenguez $\mathrm{P}$ et al (1995). The role of mesolimbic dopaminergic and retrohippocampal afferents to the nucleus accumbens in latent inhibition: implications for schizophrenia. Behav Brain Res 71: 19-31.

Gray JA, Moran PM, Grigoryan G, Peters SL, Young AM, Joseph MH (1997). Latent inhibition: the nucleus accumbens connection revisited. Behav Brain Res 88: 27-34.

Green MF, Kern RS, Braff DL, Mintz J (2000). Neurocognitive deficits and functional outcome in schizophrenia: are we measuring the 'right stuff? Schizophr Bull 26: 119-136.

Gur RE, Jaggi JL, Shtasel DL, Ragland JD, Gur RC (1994). Cerebral blood flow in schizophrenia: effects of memory processing on regional activation. Biol Psychiatry 35: 3-15.

Heckers S, Curran T, Goff D, Rauch SL, Fischman AJ, Alpert NM et al (2000). Abnormalities in the thalamus and prefrontal cortex during episodic object recognition in schizophrenia. Biol Psychiatry 48: 651-657.

Heckers S, Rauch SL, Goff D, Savage CR, Schacter DL, Fischman AJ et al (1998). Impaired recruitment of the hippocampus during conscious recollection in schizophrenia. Nat Neurosci 1: 318-323.

Hemsley DR (1994). A cognitive model for schizophrenia and its possible neural basis. Acta Psychiatr Scand Suppl 384: 80-86.

Jakob H, Beckmann H (1986). Prenatal developmental disturbances in the limbic allocortex in schizophrenics. J Neural Transm 65: 303-326.

Johnston MV, Coyle JT (1982). Cytotoxic lesions and the development of transmitter systems. Trends Neurosci 5: 153-156.

Johnston MV, Barks J, Greenamyre T, Silverstein F (1988). Use of toxins to disrupt neurotransmitter circuitry in the developing brain. Prog Brain Res 73: 425-446.

Jongen-Rêlo A, Leng A, Lüber M, Pothuizen H, Weber L, Feldon J (2004). The prenatal methylazoxymethanol acetate treatment: a neurodevelopmental animal model for schizophrenia? Behav Brain Res 149: 159-181.

Kosmidis MH, Breier A, Fantie BD (1999). Avoidance learning in schizophrenia: a dissociation between the effects of aversive and non-aversive stimuli. Schizophr Res 38: 51-59.

Kovelman JA, Scheibel AB (1984). A neurohistological correlate of schizophrenia. Biol Psychiatry 19: 1601-1621.

Lacroix L, White I, Feldon J (2002). Effect of excitotoxic lesions of rat medial prefrontal cortex on spatial memory. Behav Brain Res 133: 69-81.

Le Pen G, Gaudet L, Mortas P, Mory R, Moreau JL (2002). Deficits in reward sensitivity in a neurodevelopmental rat model of schizophrenia. Psychopharmacology 161: 434-441.
Lipska BK, Jaskiw GE, Weinberger DR (1993). Postpubertal emergence of hyperresponsiveness to stress and to amphetamine after neonatal excitotoxic hippocampal damage: a potential animal model of schizophrenia. Neuropsychopharmacology 9: 67-75.

Manoach DS (2003). Prefrontal cortex dysfunction during working memory performance in schizophrenia: reconciling discrepant findings. Schizophr Res 60: 285-298.

Mednick SA, Machon RA, Huttunen MO, Bonett D (1988). Adult schizophrenia following prenatal exposure to an influenza epidemic. Arch Gen Psychiatry 45: 189-192.

Moore H, Grace AA (1997). Anatomical changes in limbic structures produced by methylazoxymethanol acetate (MAM) during brain development are associated with changes in physiological interactions among afferents to the nucleus accumbens. Soc Neurosci Abstr 23: 2378.

Moritz S, Krausz M, Gottwalz E, Lambert M, Perro C, Ganzer S et al (2000). Cognitive dysfunction at baseline predicts symptomatic 1-year outcome in first-episode schizophrenics. Psychopathology 33: $48-51$

Morris R (1984). Developments of a water-maze procedure for studying spatial learning in the rat. J Neurosci Methods 11: 47-60.

Morris RG, Garrud P, Rawlins JN, O’Keefe J (1982). Place navigation impaired in rats with hippocampal lesions. Nature 297: 681-683.

Moser E, Moser MB, Andersen P (1993). Spatial learning impairment parallels the magnitude of dorsal hippocampal lesions, but is hardly present following ventral lesions. J Neurosci 13: 3916-3925.

Porsolt RD, Anton G, Blavet N, Jalfre M (1978). Behavioural despair in rats: a new model sensitive to antidepressant treatments. Eur J Pharmacol 47: 379-391.

Ragland JD, Gur RC, Glahn DC, Censits DM, Smith RJ, Lazarev MG et al (1998). Frontotemporal cerebral blood flow change during executive and declarative memory tasks in schizophrenia: a positron emission tomography study. Neuropsychology 12: 399-413.

Ragland JD, Gur RC, Raz J, Schroeder L, Kohler CG, Smith RJ et al (2001). Effect of schizophrenia on frontotemporal activity during word encoding and recognition: a PET cerebral blood flow study. Am J Psychiatry 158: 1114-1125.

Ravard S, Carnoy P, Herve D, Tassin JP, Thiebot MH, Soubrie P (1990). Involvement of prefrontal dopamine neurones in behavioural blockade induced by controllable $v s$ uncontrollable negative events in rats. Behav Brain Res 37: 9-18.

Sams-Dodd F, Lipska BK, Weinberger DR (1997). Neonatal lesions of the rat ventral hippocampus result in hyperlocomotion and deficits in social behaviour in adulthood. Psychopharmacology 132: 303-310.

Schacter DL, Reiman E, Uecker A, Polster MR, Yun LS, Cooper LA (1995). Brain regions associated with retrieval of structurally coherent visual information. Nature 376: 587-590.

Schacter DL, Uecker A, Reiman E, Yun LS, Bandy D, Chen K et al (1997). Effects of size and orientation change on hippocampal activation during episodic recognition: a PET study. Neuroreport 8: 3993-3998.

Siegel S (1956). Nonparametric Statistics for the Behavioral Sciences. McGraw-Hill: New York.

Stark H, Bischof A, Wagner T, Scheich H (2000). Stages of avoidance strategy formation in gerbils are correlated with dopaminergic transmission activity. Eur J Pharmacol 405: 263-275.

Stark H, Bischof A, Wagner T, Scheich H (2001). Activation of the dopaminergic system of medial prefrontal cortex of gerbils during formation of relevant associations for the avoidance strategy in the shuttle-box. Prog Neuropsychopharmacol Biol Psychiatry 25: 409-426. 
Stark H, Rothe T, Wagner T, Scheich H (2004). Learning a new behavioral strategy in the shuttle-box increases prefrontal dopamine. Neuroscience 126: 21-29.

Susser E, Neugebauer R, Hoek HW, Brown AS, Lin S, Labovitz D et al (1996). Schizophrenia after prenatal famine. Further evidence. Arch Gen Psychiatry 53: 25-31.

Talamini LM, Koch T, Luiten PG, Koolhaas JM, Korf J (1999). Interruptions of early cortical development affect limbic association areas and social behaviour in rats; possible relevance for neurodevelopmental disorders. Brain Res 847: 105-120.

Tucci S, Fernandez R, Baptista T, Murzi E, Hernandez L (1994). Dopamine increase in the prefrontal cortex correlates with reversal of haloperidol-induced catalepsy in rats. Brain Res Bull 35: 125-133.

van Haaren F, de Bruin JP, Heinsbroek RP, Van de Poll NE (1985). Delayed spatial response alternation: effects of delay-interval duration and lesions of the medial prefrontal cortex on

response accuracy of male and female Wistar rats. Behav Brain Res 18: 41-49.

van Haaren F, van Zijderveld G, van Hest A, de Bruin JP, van Eden CG, Van de Poll NE (1988). Acquisition of conditional associations and operant delayed spatial response alternation: effects of lesions in the medial prefrontal cortex. Behav Neurosci 102: 481-488.

Virgili M, Barnabei O, Contestabile A (1989). Regional- and agespecific neurochemical alterations in rats rendered microencephalic by differentially timed gestational methylazoxymethanol treatment. Eur J Neurosci 1: 647-653.

West AP (1990). Neurobehavioral studies of forced swimming: the role of learning and memory in the forced swim test. Prog Neuropsychopharmacol Biol Psychiatry 14: 863-877.

Zornberg GL, Buka SL, Tsuang MT (2000). Hypoxicischemiarelated fetal/neonatal complications and risk of schizophrenia and other nonaffective psychoses: a 19-year longitudinal study. Am J Psychiatry 157: 196-202. 